\title{
Chromosome number of Camellia changii Ye
}

\author{
Tsuyoshi Ito ${ }^{1}$ and Katsuhiko Kondo ${ }^{2}$ \\ ${ }^{1}$ Botanical Garden, Atsugi Farm, Tokyo University of Agriculture, \\ 1737 Funako, Atsugi City 243-0034, Japan; \\ ${ }^{2}$ Laboratory of Plant Genetics and Breeding Science, Department of Agriculture, \\ Faculty of Agriculture, Tokyo University of Agriculture, \\ 1737 Funako, Atsugi City 243-0034, Japan
}

${ }^{2}$ Author for correspondence: (k3kondo@nodai.ac.jp) Received June 5, 2010; accepted September 27, 2010

\begin{abstract}
The chromosome number of Camellia changii Ye, a summer flowering camellia, was 2n=30, diploid (X=15), reported here for the first time.
\end{abstract}

KEYWORDS: Camellia changii, Chromosome number, Diffuse type resting nucleus, Diploid (2n=30)

Camellia changii Ye was discovered in a small area in a hilly area altitude at $300-450 \mathrm{~m}$ in Yangchun City, Guangdong Province, the People's Republic of China (Cao et al. 2005). It was described by Ye (1985) rather new to Camellia after a long time people thought it would be an azalea (see Fig. 1) since this species was very unusual to the genus to bloom during the mid-summer although most of the species of Camellia flower during the autumn, winter to spring seasons in the natural distribution in the northern hemisphere. It looks like Rhododendron in flowering season, flower and leaf morphology (Fig. 1).

Chromosome numbers of Camellia have been reported by Ackerman (1971), Kondo (1977, 1978a, 1990). However, some species of the genus have been reported no chromosome counts due to some reasons such as newly discovered or described taxa. They must be placed in the basic chromosome number of $\mathrm{X}=15$ (Ackerman 1971; Kondo 1977, 1978a, 1990). Reporting chromosome numbers is very important to get a basic information on close relationships of species to make artificial crosshybridization.

The chromosome number of $C$. changii is here reported for the first time.

\section{Materials and Methods}

Plant material. Camellia changii Ye (Fig. 1) was currently introduced at commercial base including to Japanese markets. A grafted individual plant of the species was purchased and made stem-cuttings for the purpose of chromosome counting.

Methods. Root tips of the plant of the species were harvested and placed in $0.002 \mathrm{M} 8$-oxyquinoline at room temperature for $2 \mathrm{~h}$. They were fixed in $45 \%$ acetic acid at $4^{\circ} \mathrm{C}$ for $5 \mathrm{~min}$ before they were macerated in $1 \mathrm{~N} \mathrm{HCl}$ : $45 \%$ acetic acid $=2: 1$ at $60^{\circ} \mathrm{C}$ for $10 \mathrm{sec}$. Their root-tips were cut and stained in 1\% aceto-orcein on glass slides

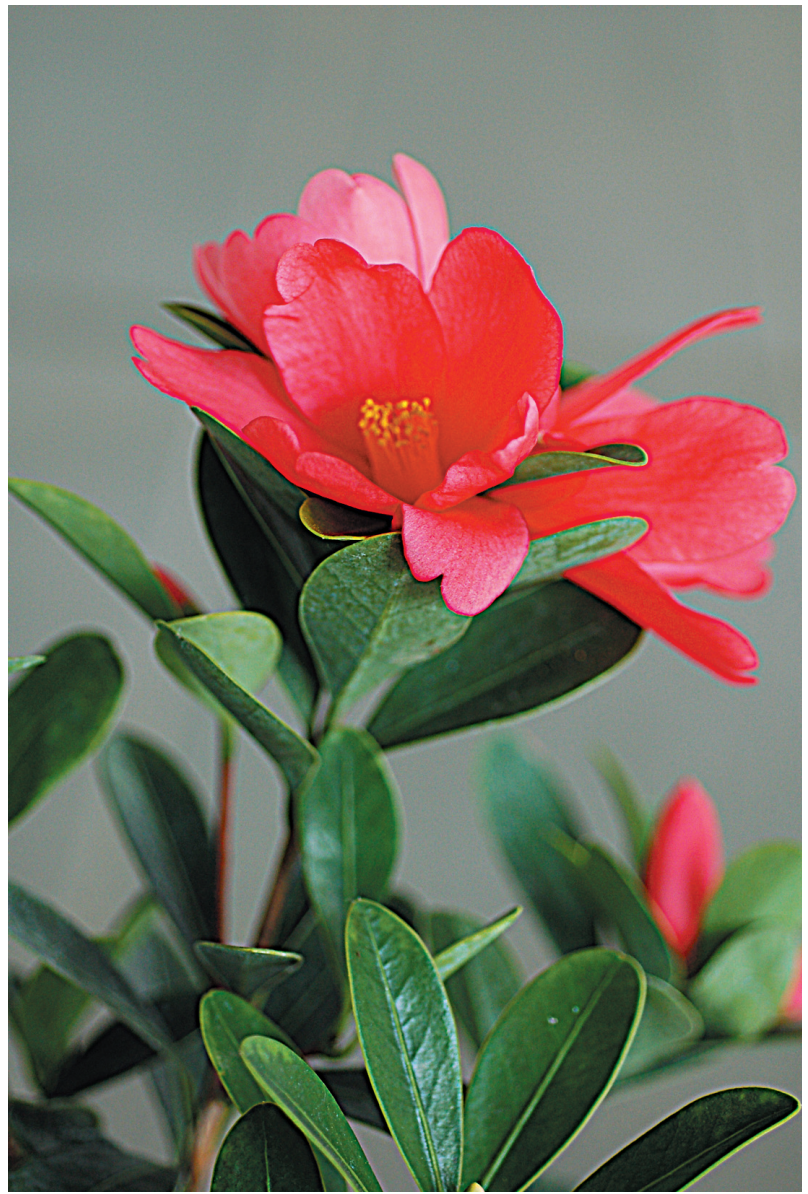

Fig. 1. Flowers and leaves of Camellia changii Ye

and were in moistened chamber for $2 \mathrm{~h}$. Then, they were put cover-slips, tapped and squashed. They were, then, pressed well by fingers and sealed by paper bonds.

\section{Results And Discussion}

Camellia changii Ye had the resting nucleus of the diffuse type (Fig. 2a), which was same as that of the other species studied (Kondo 1978b). The chromosome number of the species was $2 n=30$ (diploid), that was reported here for 


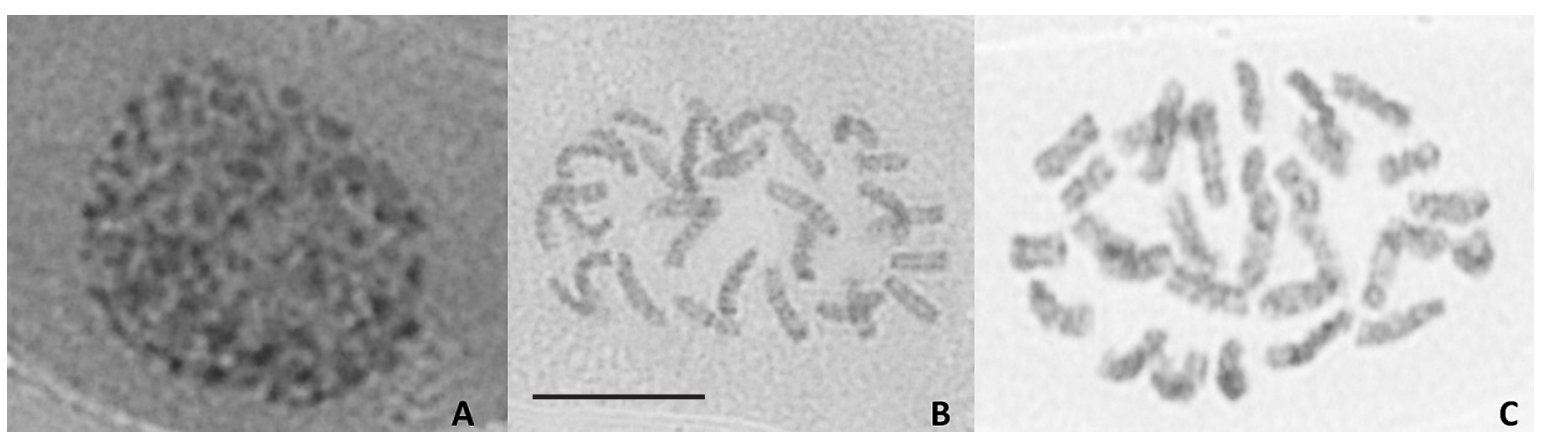

Fig. 2. Karyomorphology of Camellia changii Ye. A. Diffuse type of resting nucleus. B. 2 n=30 at prometaphase. C. $2 \mathrm{n}=30$ at metaphase (diploid). Bar $=4.0 \mu \mathrm{m}$

the first time (Fig. 2b and c).

Camellia changii has the most spectacular habit on summer-flowering that has never been found in any other species of the genus. It was thought as an azalea for a long time due to morphology of flowers and leaves and flowering season of some species of evergreen azaleas. The summer flowering habit of $C$. changii should be introduced to the cultivation world of Camellia by artificial cross breeding shortly to see Camellia flowers from the winter-limited period to all-year around flowering seasons.

ACKNOWLEDGMENTS. This study was supported by the Grantin-Aid for Scientific Research Program (A)(I) 19255004 of Japan Society for the Promotion of Science (Representative: Katsuhiko Kondo).

\section{Literature Cited}

Ackerman, W. L. 1971. Genetic and cytological studies with Camellia and related genera. Tech. Bull. No. 1427. Agricul. Res. Service, USDA, U.S. Govern. Print. Office, Washington, D.C. pp. 115

Cao, J., Parks, C. R. and Du Y. 2005. Collected species of the genus Camellia an illustrated outline. Zhejiang Science and Technology Press, p. 302.

Gu, Z., Xia, L., Xie L. and Kondo, K. 1988. Report on the chromosome numbers of some species of Camellia in China. Acta Bot. Yunnanica 10: 291-296.

Kondo, K. 1977. Chromosome numbers in the genus Camellia. Biotropica 9: 86-94.

Kondo, K. 1978a. Chromosome numbers of certain Camellia species and related genera, p. 334-347. In: D. L. Feathers and M. L. Brown, Eds., The Camellia. Am. Camellia Soc., The R. L. Bryan Co., Columbia, S.C., U.S.A., pp. 476.

Kondo, K. 1978b. Cytological studies in cultivated species of Camellia. Shin-Kaki 99: 41-53 (in Japanese).

Kondo, K. 1990. Chromosome numbers of some Chinese species of Camellia. Bulletin of Seibu Maizuru Botanical Institute 5: 1-9

Ye, C. 1985. A new species of Camellia in Guangdong. Bull. Guangdong Bot. Assoc. 1985 (1): 3 (in Chinese) 